\title{
The analytic hierarchy process: can wash criteria be ignored?
}

\author{
J.S. Finan ${ }^{\mathrm{a}}$, W.J. Hurley ${ }^{\mathrm{b}, *}$ \\ ${ }^{a}$ Department of Political and Economic Science, Royal Military College of Canada, CP 17000, \\ Succursale Forces, Kingston, Ont., Canada K7K $7 B 4$ \\ ${ }^{\mathrm{b}}$ Department of Business Administration, Royal Military College of Canada, CP 17000, \\ Succursale Forces, Kingston, Ont., Canada K7K 7B4
}

Received 1 January 2000; received in revised form 1 August 2000

\begin{abstract}
We define a wash criterion as one where the decision-maker is indifferent among the alternatives when they are compared on that criterion. In view of the Belton-Gear example and other such anomalies associated with the analytic hierarchy process (AHP), we ask whether eliminating a wash criterion will affect the overall ranking of objects. In the case where there is only one level of criteria, the rank-order of objects is unaffected by leaving out a wash criterion. However, in the case where the wash criterion is a subcriterion, the rank order may be affected by leaving it out.
\end{abstract}

\section{Scope and purpose}

A wash criterion is defined as a criterion where the decision-maker is indifferent among the alternatives when they are compared on that criterion. We would like to think that the overall rank-order of objects would be unaffected in the case where the wash criterion is excluded. We give an example of an AHP hierarchy where this is not the case. In our view this presents another challenge to the AHP methodology. (C) 2002 Elsevier Science Ltd. All rights reserved.

Keywords: Multicriteria decision analysis; AHP; Belton-Gear

\section{Introduction}

In the case where the analytic hierarchy process (AHP, see Saaty [1-3]) is applied to a multicriteria decision, we define a wash criterion as one where the decision-maker (DM) is indifferent

\footnotetext{
* Corresponding author. Tel.: + 1-613-541-6000 (Ext: 6468); fax: + 1-613-541-6315.

E-mail address: hurley_w@rmc.ca (W.J. Hurley).
} 
among the alternatives when they are compared on that criterion. This type of criterion is sometimes termed a non-discriminating criterion. In view of the Belton-Gear [4] example and other such anomalies associated with the AHP, we examine whether eliminating a wash criterion will affect the overall ranking of objects.

In our experience, this problem has come up in a variety of contexts. One example concerned the choice of strategic direction for an integrated oil and gas firm in Western Canada. Senior management felt that it was important that the firm grow in order to remain competitive. There were three options: buy a large competitor; buy a small competitor; and the status quo (grow as fast as internally generated funds would allow). There were four criteria, and of these, one of the most important was earnings per share. But when the numbers were run, these three options produced an identical earnings per share. The analyst concluded that earnings per share was a wash criterion and eliminated it. Subsequently, the three options were assessed on the three remaining criteria.

Here is another example. Some years ago the Canadian forces were interested in purchasing an unmanned battlefield surveillance system. One of the criteria was mission survivability - the probability the vehicle would survive a well-defined average scenario. The manufacturers' glossies all estimated this survival probability to be 0.90 give or take a couple of percent depending on the manufacturer. Given that the decision exercise was an initial screening (the top three to four moved on), and there was no way to differentiate the manufacturers on this criterion, our base assumption was that mission survivability was a wash criterion.

We consider a general AHP hierarchy in the case where a DM is trying to rank-order the alternatives. We denote an AHP hierarchy where there are $t$ levels of criteria as $H(t)$. Hence $H(1)$ is a hierarchy with only one level of criteria; $H(2)$ is a hierarchy with subcriteria. In view of the popularity of the multiplicative AHP (see Barzilai and Golany [5], Barzilai [6], and Barzilai et al. [7]), we consider two schemes for collapsing the hierarchy into an overall set of weights: one is the additive or Saaty method (SAHP); the other is the multiplicative procedure (MAHP).

We show the following results. In the case where there is only one level of criteria and the DM is perfectly consistent, the rank-order of objects is unaffected by leaving out a wash criterion regardless of which evaluation procedure is used. However, in the case where the wash criterion is a subcriterion, the rank-order may be affected by leaving it out.

\section{Proof that $H(1)$ wash criteria are irrelevant}

Suppose the DM begins with $n+1$ criteria indexed by the set $J=\{0,1, \ldots, n\}$ and $m$ choice alternatives indexed by $I=\{1,2, \ldots, m\}$. The DM's problem is to determine a rank-order of the $m$ alternatives. The wash criterion is indexed by 0 . We index the reduced set of criteria by $\bar{J}=\{1, \ldots, n\}$. Note that, as defined, this is an $H(1)$ hierarchy.

We assume the DM is perfectly consistent. Denote the set of weights for the full criteria set $J$ by $c_{j}$, and for the reduced criteria set by $\bar{c}_{j}$. Then we have that

$$
c_{j}=\left(1-c_{0}\right) \bar{c}_{j} \quad \text { for } j=1,2, \ldots, n .
$$


To see this, suppose the elements of the pairwise comparison matrix for the full criteria set has elements $a_{i j}$ and note that

$$
\frac{c_{i}}{c_{j}}=a_{i j}=\frac{\bar{c}_{i}}{\bar{c}_{j}} \text { for all } i, j \geqslant 1 .
$$

Note that, under the assumption in (2.1), the set of weights for the full criteria set sums to 1:

$$
\begin{aligned}
\sum_{i=0}^{m} c_{i} & =c_{0}+\sum_{i=1}^{m}\left(1-c_{0}\right) \bar{c}_{i} \\
& =c_{0}+\left(1-c_{0}\right) \sum_{i=1}^{m} \bar{c}_{i} \\
& =1 \quad \text { since } \sum_{i=1}^{m} \bar{c}_{i}=1 .
\end{aligned}
$$

Let $u_{i j}$ be the weight of alternative $i$ measured on criterion $j$ assuming that the SAHP evaluation procedure is used. Then $\sum_{i} u_{i j}=1$ for all $j$. In particular, we have that

$$
u_{i 0}=\frac{1}{m} \text { for all } i \text {. }
$$

Let the SAHP overall weights of the alternatives for the full criteria set be denoted $w_{i}^{+}$, and for the reduced criteria set, $\bar{w}_{i}^{+}$. We now show that rank-order of alternatives is unaffected by eliminating the wash criterion.

Proposition 1. $\bar{w}_{i}^{+} \gtreqless \bar{w}_{j}^{+} \Leftrightarrow w_{i}^{+} \gtreqless w_{j}^{+}$for all $i, j \in I$.

Proof. The overall weight for alternative $i$ over the reduced set is

$$
\bar{w}_{i}^{+}=\sum_{k} \bar{c}_{k} u_{i k},
$$

and for alternative $j$

$$
\bar{w}_{j}^{+}=\sum_{k} \bar{c}_{k} u_{j k} .
$$

Taking the difference, we have

$$
\bar{w}_{i}^{+}-\bar{w}_{j}^{+}=\sum_{k} \bar{c}_{k} u_{i k}-\sum_{k} \bar{c}_{k} u_{j k} .
$$

Now examine $w_{i}^{+}-w_{j}^{+}$:

$$
w_{i}^{+}-w_{j}^{+}=\sum_{k} c_{k} u_{i k}-\sum_{k} c_{k} u_{j k}
$$




$$
\begin{aligned}
& =\frac{1}{m} c_{0}+\sum_{k}\left(1-c_{0}\right) \bar{c}_{k} u_{i k}-\frac{1}{m} c_{0}-\sum_{k}\left(1-c_{0}\right) \bar{c}_{k} u_{j k} \\
& =\left(1-c_{0}\right)\left\{\sum_{k} \bar{c}_{k} u_{i k}-\sum_{k} \bar{c}_{k} u_{j k}\right\} \\
& =\left(1-c_{0}\right)\left\{\bar{w}_{i}^{+}-\bar{w}_{j}^{+}\right\} .
\end{aligned}
$$

Hence, the sign of $w_{i}^{+}-w_{j}^{+}$is the same as the sign of $\bar{w}_{i}^{+}-\bar{w}_{j}^{+}$and the proof is complete.

The conclusion is that the rank-order of alternatives in an $H(1)$ hierarchy is unaffected by eliminating a wash criterion in the case where the SAHP evaluation is used. This result is easily extended to the case where there are a number of wash criteria. It also extends to the case where the MAHP evaluation procedure is used, as we now show.

Let $v_{i j}$ be the weight of alternative $i$ measured on criterion $j$ assuming that the MAHP evaluation procedure is used. Then $\prod_{i} v_{i j}=1$ for all $j$. In particular, we have

$$
v_{i 0}=1 \text { for all } i \text {. }
$$

Let the MAHP overall weights for the full criteria set be denoted $w_{i}^{\times}$, and for the reduced criteria set, $\bar{w}_{i}^{\times}$.

Proposition 2. $\bar{w}_{i}^{\times} \gtreqless \bar{w}_{j}^{\times} \Leftrightarrow w_{i}^{\times} \gtreqless w_{j}^{\times}$for all $i, j \in I$.

Proof. The overall weight for alternative $i$ over the reduced set is

$$
\bar{w}_{i}^{\times}=v_{i 1}^{\bar{c}_{1}} v_{i 2}^{\bar{c}_{2}} \ldots v_{i m}^{\bar{c}_{m}}
$$

Over the full set, it is

$$
\begin{aligned}
w_{i}^{\times} & =1^{c_{0}} v_{i 1}^{c_{1}} v_{i 2}^{c_{2}} \ldots v_{i m}^{c_{m}} \\
& =v_{i 1}^{\left(1-c_{0}\right) \bar{c}_{1}} v_{i 2}^{\left(1-c_{0}\right) \bar{c}_{2}} \ldots v_{i m}^{\left(1-c_{0}\right) \bar{c}_{m}} \\
& =\left(v_{i 1}^{\bar{c}_{1}} v_{i 2}^{\bar{c}_{2}} \ldots v_{i m}^{\bar{c}_{m}}\right)^{1-c_{0}} \\
& =\left(\bar{w}_{i}^{\times}\right)^{1-c_{0}} .
\end{aligned}
$$

Therefore, we have

$$
w_{i}^{\times}=\left(\bar{w}_{i}^{\times}\right)^{1-c_{0}}
$$

and the result of the proposition follows directly.

Hence, Propositions 1 and 2 demonstrate that, regardless of the evaluation scheme, the rankorder of objects in an $H(1)$ hierarchy is unaffected by ignoring a wash criterion. It is important to note that our general result that wash criteria can be ignored in a hierarchy with one level of criteria depends critically on the DM being perfectly consistent. We cannot prove the same result in the case of an imperfectly consistent DM. 


\section{What about wash subcriteria?}

Consider the following $H(2)$ hierarchy:

Goal

Main criteria

Main criteria weights

Subcriteria

Subcriteria weights

Option $A_{1}$

Option $A_{2}$
G

$J \quad J^{\prime}$

0.55

0.45

where $J_{0}$ is the wash subcriterion. The following table gives the overall SAHP weights of $A_{1}$ and $A_{2}$ in two cases: one where $J_{0}$ is included and the other where it is not:

$\begin{array}{lll} & \text { With } J_{0} & \text { With } \\ \text { Weight of } A_{1} & 0.477 & 0.51 \\ \text { Weight of } A_{2} & 0.523 & 0.49\end{array}$

Note that, with $J_{0}, A_{2}$ is preferred to $A_{1}$, and in the case where $J_{0}$ is left out $A_{1}$ is preferred to $A_{2}$. Hence this simple example demonstrates that wash subcriteria cannot be ignored when the SAHP evaluation procedure is used.

But the MAHP is no better. If the MAHP is applied to this same hierarchy, we get the following weights:

$\begin{array}{lll} & \text { With } J_{0} & \text { With } \\ \text { Weight of } A_{1} & 0.967 & 1.050 \\ \text { Weight of } A_{2} & 1.034 & 0.952\end{array}$

And again note the reversal with and without the wash criterion.

\section{Conclusion}

Our results have the flavour of the Belton-Gear example. We would like to think that the overall rank-order of objects should be unaffected by including or excluding wash criteria. But this is not the case. While it is true that, for a hierarchy with a single level of criteria, that rank-order is unaffected, the same does not hold for hierarchies with multiple levels of criteria. Even the MAHP technique for computing the overall weights does not work in this latter case. In view of the fact that every hierarchy with multiple levels of criteria can, in principle, be modelled as a hierarchy with a single level of criteria, it must be that our methods for collapsing a hierarchy with multiple 
levels of criteria are incorrect. In sum, we view our results as a serious challenge to the AHP methodology.

\section{Acknowledgements}

We would like to thank two anonymous referees for very helpful comments.

\section{References}

[1] Saaty TL. A scaling method for priorities in hierarchical structures. Journal of Mathematical Psychology 1977;15:234-81.

[2] Saaty TL. The analytic hierarchy process. New York: McGraw-Hill, 1980.

[3] Saaty TL. The analytic hierarchy process: what it is and how it is used. Mathematical Modelling 1987;9:161-76.

[4] Belton V, Gear T. On a shortcoming of Saaty's method of analytic hierarchies. Omega 1983;11:228-30.

[5] Barzilai J, Golany B. Deriving weights from pairwise comparison matrices: the additive case. Operations Research Letters 1990;9:407-10.

[6] Barzilai J. On the use of the eigenvector in the AHP. Proceedings of the Tenth International Conference on Multiple Criteria Decision Making, vol. 1, 1992. p. 291-300.

[7] Barzilai J, Cook WD, Golany B. The analytic hierarchy process: structure of the problem and its solutions. In: Phillips FY, Rousseau JJ, editors. Systems and management science by extremal methods. Dordrecht: Kluwer Academic Publishers, 1992, p. 361-371.

W.J. Hurley is a Professor in the Department of Business Administration at the Royal Military College of Canada. His research interests are decision analysis, game theory, and transportation modeling.

J.S. Finan is a Professor in the Department of Politics and Economics at the Royal Military College of Canada. His primary research interest is decision analysis and the application of decision analytic techniques to problems in international politics. 